\title{
Clonidina Associada à Morfina e Midazolam em Crianças Submetidas à Ventilação Mecânica: Estudo Aleatório, Duplamente Encoberto e Placebo Controlado*
}

\author{
Clonidine Associated to Morphine and Midazolam \\ in Children Submitted to Mechanical Ventilation: \\ Randomized, Double Blind and Placebo Controlled Study
}

Marizete Molon ${ }^{1}$, Jefferson Piva², Patrícia Karcher ${ }^{3}$, Trudiane Baldissera ${ }^{4}$

\section{RESUMO}

JUSTIFICATIVA E OBJETIVOS: A síndrome de abstinência tem alta prevalência em nosso meio e, além do desconforto, prolonga o tempo de internação. O objetivo deste estudo foi avaliar a influência da adição de clonidina por via oral, à infusão contínua de morfina e midazolam na sedação de crianças submetidas à ventilação mecânica.

MÉTODO: Foi realizado um estudo aleatório, duplamente encoberto e placebo controlado na UTI Pediátrica do Hospital Geral de Caxias do Sul, no período de maio de 2005 a agosto de 2006. Foram Incluídas crianças com idade entre 1 e 36 meses, submetidas à ventilação mecânica por mais de 12 horas, que utilizaram morfina e midazolam em infusão contínua. Foram divididas em dois grupos: clonidina $(5 \mu \mathrm{g} / \mathrm{kg})$ e placebo, associados à infusão de morfina e midazolam. As doses dos sedativos em infusão contínua foram definidas pelos médicos

1. Mestre em Pediatria e Ciências Aplicadas a Pediatria pela UFRGS. Médica Intensivista da UTI Pediátrica do Hospital Geral de Caxias do Sul.

2. Professor adjunto do Departamento de Pediatria da FM-UFRGS. Professor Adjunto do Departamento de Pediatria da FM-PUCRS. Chefe associado da UTIP do Hospital São Lucas da PUCRS.

3. Residente de Terceiro Ano em Terapia Intensiva Pediátrica do Hospital Geral de Caxias do Sul

4. Residente de Segundo Ano em Pediatria do Hospital Geral de Caxias do Sul.

${ }^{*}$ Recebido da Unidade de Terapia Intensiva Pediátrica do Hospital Geral de Caxias do Sul, Caxias do Sul, RS

Apresentado em 08 de maio de 2007

Aceito para publicação em 19 de julho de 2007

Endereço para correspondência:

Dra. Marizete E. Molon

Rua Sinimbú 2211, Sala 310

95050-310 Caxias do Sul, RS

E-mail: marimolon@terra.com.br

(C)Associação de Medicina Intensiva Brasileira, 2007 assistentes. Diariamente foram registradas as doses de sedativos administradas nas 24 horas, assim como as doses intermitentes e aplicado o escore de Finnegan para definir e quantificar abstinência. Os grupos foram comparados quanto às doses de sedativos administradas, tempo de uso de infusão contínua, presença e duração da abstinência.

RESULTADOS: Foram incluídas 69 crianças (31 no grupo clonidina e 38 no placebo). Os grupos foram semelhantes nas características gerais (peso, idade, sexo, indicação de ventilação mecânica). Ambos os grupos utilizaram altas doses cumulativas de sedativos, tanto em infusão contínua quanto intermitente. Completaram o estudo 59 pacientes, 25 no grupo clonidina e 34 no placebo. Em ambos os grupos a prevalência da abstinência foi semeIhante (72\% e $75 \%$, respectivamente), sem diferença na sua duração e no tempo de ventilação mecânica.

CONCLUSÕES: Em crianças submetidas à ventilação mecânica que utilizaram altas doses de opióides e diazepínicos, a adição de clonidina não promoveu redução nas doses diárias ou cumulativas de sedativos, não influiu na prevalência ou na evolução da abstinência.

Unitermos: abstinência, clonidina, sedação, ventilação mecânica

\section{SUMMARY}

BACKGROUND AND OBJECTIVES: In our country, the abstinence syndrome has high prevalence and besides the distress prolongs the length of hospital stay. The aim of this study was to evaluate the influence of associating oral clonidine to the intravenous morphine plus midazolam continuous infusion in children submitted to mechanical ventilation.

METHODS: Randomized, double blind, placebo controlled study, conducted in the PICU at the Hospital Geral 
of Caxias do Sul. All children (1-36 months) submitted to mechanical ventilation over 12 hours (May-2005/August2006), which had used intravenous morphine and midazolam continuous infusion were included. They were randomized to received clonidine $(5 \mu \mathrm{g} / \mathrm{kg})$ or placebo associated to the sedative continuous infusion. The infusion sedative doses were at the discretion of the assistant physician. The administered doses in the previous 24 hours and the doses of intermittent sedation were daily collected. The abstinence syndrome was defined based on Finnegan Score. The groups were compared regarding the cumulative doses of sedatives, length of use of sedative continuous infusion, presence and duration of the abstinence.

RESULTS: Were included 69 patients (31 in the clonidine group and 38 in the placebo group). The two groups were similar regarding the general characteristics (weight, age, gender, indication of mechanical ventilation). The midazolam and morphine doses (cumulative and intermittent doses) were not different in both groups. Completed the study 59 patients, 25 in clonidine group and 34 in placebo group. The prevalence of the abstinence was similar $(72 \%$ and $75 \%$, respectively), without difference in the recovery time neither related to the length of mechanical ventilation.

CONCLUSIONS: In children submitted to mechanical ventilation using high dose of opioids and diazepinic infusion the addiction of clonidine did not decrease the daily doses neither the cumulative doses and nevertheless reduced the prevalence and the evolution of abstinence syndrome.

Key Words: abstinence, clonidine, mechanical ventilation, sedation

\section{INTRODUÇÃO}

Até recentemente acreditava-se que as crianças não sentiam dor, por isso não recebiam sedativos ou analgésicos durante a ventilação mecânica (VM). Posteriormente, verificou-se que múltiplos fatores contribuem para a dor e a ansiedade no paciente criticamente enfermo, especialmente quando é submetido à imobilização e VM, ou procedimentos como intubação e aspiração traqueal ${ }^{1,2}$. A profilaxia e o combate a esses sintomas são imprescindíveis, tanto do ponto de vista ético quanto pelas necessidades físicas, além de comprovadamente auxiliar na recuperação do paciente ${ }^{3}$.

O controle da sedação é, ainda nos dias atuais, baseado em critérios subjetivos ${ }^{1,2,4}$. Com o incremento no uso de sedativos, passou-se de um estágio inicial de subvalo- rização da sedação, quando era comum a ocorrência de agitação durante a VM, para uma fase de supervalorização da analgesia e sedação, em que os pacientes são mantidos com sedação máxima. A excessiva sedação utilizada comumente em crianças em VM aumenta a incidência de hipotensão, o tempo de ventilação mecânica e a ocorrência de tolerância e abstinência ${ }^{1-3}$. Com o intuito de promover boa sedação e mínima abstinência, novos sedativos têm sido preconizados. Dentre as medicações utilizadas com essa finalidade está a clonidina $^{5-7}$. A clonidina é um $\alpha_{2}$-agonista de ação central, usado inicialmente no tratamento da hipertensão, porém com propriedades sedativas e analgésicas. Os receptores $\alpha_{2}$-adrenérgicos têm parte de sua ação farmacológica através da ativação dos mesmos canais de potássio que os receptores opióides $2,5,8-13$.

A associação de clonidina ao esquema de retirada do opióide pode reduzir os sinais de agitação, choro, salivação, tremores, diarréia, dificuldade de alimentação e, inclusive o tempo de hospitalização 2,8,12,14-17. Alguns estudos também demonstraram que a clonidina, quando adicionada ao esquema de sedação, pode potencializar tanto a sedação quanto a analgesia ${ }^{2,9,10,12-15,18-26,28,33} . \mathrm{Na}$ maioria destes estudos a clonidina foi associada a baixas doses de opióides e diazepínicos. Entretanto, em nosso meio, a sedação e analgesia de crianças submetidas à ventilação mecânica se caracteriza por uso de altas doses de opióides e diazepínicos ${ }^{1,4}$. Portanto, existem dúvidas se a clonidina teria o mesmo efeito na prevenção da síndrome de abstinência quando associada à doses maiores de opióides e diazepínicos.

O objetivo deste estudo foi avaliar o efeito da adição de clonidina em crianças submetidas à ventilação mecânica, que utilizaram doses elevadas de morfina e midazolam em infusão contínua. A hipótese é que a adição de clonidina possa modificar as doses necessárias dos sedativo-analgésicos utilizados, o tempo de utilização, a prevalência e a duração da abstinência.

\section{MÉTODO}

Após aprovação das Comissões Científica e de Ética, foi realizado ensaio clínico, aleatório, duplamente encoberto e placebo controlado na UTI Pediátrica do Hospital Geral de Caxias do Sul. A exigência da assinatura do termo de consentimento por parte dos pais ou responsáveis foi cumprida.

A UTI Pediátrica (UTIP) desse hospital tem capacidade de 9 leitos, com média de admissões de 20 crianças/ mês, sendo que a proporção de crianças em ventila- 
ção mecânica (VM) varia de $70 \%$ a $100 \%$, com mediana de permanência em VM de sete dias. A equipe médica dessa UTIP é composta por médicos assistentes, rotineiros, residentes e plantonistas. A estratégia consensual desse grupo é que a sedação de crianças em VM seja feita prioritariamente por meio de infusão continua de morfina e midazolam, visando mantê-las com mínima movimentação durante a fase aguda da doença (equivalente a 4 na escala de Ramsay; ou, sedação profunda). Para tal fim, de acordo com o critério médico assistente ou plantonista, permite-se que a velocidade de infusão seja aumentada ou que doses suplementares sejam administradas.

Foram incluídas no estudo todas as crianças com idade entre um e 36 meses, submetidas à ventilação mecânica por mais de 12 horas, no período compreendido entre maio de 2005 e agosto de 2006. Todas as crianças foram submetidas à intubação traqueal e utilizaram morfina e/ou midazolam em infusão contínua, além de terem recebido sedação extra com outros fármacos ou bloqueio neuromuscular, quando necessário. Foram excluídas do estudo as crianças nas quais houve mudança para outro agente farmacológico, com descontinuidade da morfina e midazolam no esquema de sedação, quando ocorreu hipotensão arterial refratária antes da aleatorização, presença de traqueostomia, transferências ou óbitos durante o período de observação ou quando houve solicitação de exclusão pelo médico assistente.

As doses de infusão e os respectivos ajustes das doses de midazolam $(\mathrm{mg} / \mathrm{kg} / \mathrm{h})$ e morfina $(\mathrm{mg} / \mathrm{kg} / \mathrm{h})$ ficavam a critério dos médicos plantonistas, de acordo com as necessidades dos pacientes. A investigadora não participou na determinação das doses que seriam administradas e no ajuste das doses.

A classificação foi feita em blocos de dez, alocados em envelopes fechados e numerados. O sorteio ficou a cargo da farmacêutica do hospital que registrava a alocação em livro próprio para essa finalidade. As crianças foram alocadas da seguinte forma:

a) Clonidina ( $5 \mu \mathrm{g} / \mathrm{kg} /$ dose a cada $8 \mathrm{~h}$ ), diluída em xarope simples $(0,1 \mathrm{mg} / \mathrm{mL})$ por via enteral, em associação à infusão padrão da unidade com midazolam e morfina em infusão contínua.

b) Placebo (água destilada, metilparabeno, xarope simples) por via enteral, em volume correspondente à dose de clonidina a cada $8 \mathrm{~h}$. Os mesmos critérios para definição de doses em infusão de midazolam e morfina, associadas à solução placebo.

As soluções placebo e clonidina, além de volumes iguais, apresentavam o mesmo aspecto, cor e consis- tência, sendo difícil a distinção física entre eles.

Os pacientes foram avaliados diariamente por uma residente de $3^{\circ}$ ano de Terapia Intensiva Pediátrica e por uma residente de $2^{\circ}$ ano em Pediatria do Hospital, que foram especificamente treinadas na coleta de dados. Foram coletados dados gerais de todos os pacientes (idade, sexo, data da internação, inicio e fim da ventilação mecânica, diagnóstico principal, entre outros). Diariamente, às 8 horas da manhã, fazia-se a coleta dos dados do prontuário referente às doses de morfina e midazolam e somadas as doses totais administradas em $\mathrm{mg} / \mathrm{kg} / \mathrm{dia}$, acrescidas as doses intermitentes da mesma medicação. Além disto, somavam-se às doses de sedação intermitente utilizada nas 24 horas.

Como desfechos principais, os dois grupos foram comparados quanto às doses de sedativos administradas (medidas em mg/kg), o tempo de uso de infusão contínua (h) e a presença de abstinência e sua duração.

Neste estudo, abstinência foi definida através do

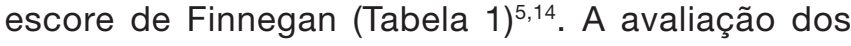

Tabela 1 - Escore de Finnegan para Avaliação de Abstinência ${ }^{7}$

\begin{tabular}{|c|c|}
\hline Sinais/Sintomas & Escores \\
\hline \multicolumn{2}{|l|}{ Choro } \\
\hline Excessivo & 2 \\
\hline Contínuo & 3 \\
\hline \multicolumn{2}{|l|}{ Sono após alimentação (h) } \\
\hline$<1$ & 3 \\
\hline$<2$ & 2 \\
\hline$<3$ & 1 \\
\hline \multicolumn{2}{|l|}{ Reflexo de Moro } \\
\hline Hiper-ativo & 2 \\
\hline \multicolumn{2}{|l|}{ Tremores } \\
\hline Leve, interrompível & 1 \\
\hline Moderado, interrompível & 2 \\
\hline Moderado, ininterrupto & 3 \\
\hline Aumento do tônus muscular & 2 \\
\hline Bocejos freqüentes & 2 \\
\hline Escoriações & 1 \\
\hline Convulsões & 5 \\
\hline Sudorese & 1 \\
\hline \multicolumn{2}{|l|}{ Febre } \\
\hline $37,8-38,3^{\circ} \mathrm{C}$ & 1 \\
\hline$>38,3^{\circ} \mathrm{C}$ & 2 \\
\hline Moteamento & 1 \\
\hline Congestão nasal & 1 \\
\hline Espirros & 1 \\
\hline Prurido nasal & 2 \\
\hline \multicolumn{2}{|l|}{ Freqüência respiratória } \\
\hline$>60 \mathrm{mov} / \mathrm{min}$ & 1 \\
\hline > 60 com retrações & 1 \\
\hline Sucção excessiva & 1 \\
\hline Alimentação deficiente & 2 \\
\hline Regurgitação & 2 \\
\hline Vômitos em jato & 3 \\
\hline \multicolumn{2}{|l|}{ Evacuações } \\
\hline Semipastosas & 2 \\
\hline Líquidas & 3 \\
\hline
\end{tabular}

Escore de 0 a 7 indica abstinência leve, de 8 a 11, moderada e 12 a 15, grave. 
escores de abstinência foi feita por profissionais especificamente treinados para este fim. Ao aplicar o escore de abstinência em 10 pacientes aleatoriamente escolhidos, essas duas pesquisadoras apresentaram um teste de concordância (Kappa) de $80 \%$. O critério de cura da síndrome de abstinência foi estabelecido como desaparecimento dos sintomas por período superior a 24 horas, sem necessidade de medicação.

Os dados dos pacientes foram transcritos para uma planilha Excel para Windows (Microsoft Office) especialmente formulada para esta pesquisa. Os dados foram analisados através do programa SPSS versão 12.0. Foi estabelecido um erro $\alpha=0,05$ e um erro $\beta=$ $20 \%$. Assumiu-se que a abstinência ocorreria em $50 \%$ das crianças e que seria considerado como significativo uma redução de $30 \%$, definindo um mínimo de 30 crianças em cada grupo.

As variáveis categóricas foram expressas em percentagem e comparadas através do teste Qui-quadrado ou risco relativo. As variáveis contínuas com distribuição normal foram expressas através de médias com o respectivo desvio-padrão e comparadas através do teste $t$ de Student. As variáveis contínuas sem distribuição normal foram expressas através de mediana com o respectivo intervalo interquartil (IQ 25\%-75\%) e comparadas através do teste de Mann-Whitney ou KruskalWallis. Foram ainda realizados testes de correlação (coeficiente de correlação de Pearson e coeficiente de determinação) entre as doses de medicamentos, tempo de tratamento da síndrome de abstinência, tempo de uso dos fármacos e tempo de tratamento da síndrome de abstinência.

\section{RESULTADOS}

Nos 15 meses de estudo (maio de 2005 a agosto de 2006), foram incluídos 69 pacientes submetidos à ventilação mecânica, tendo sido alocados 31 no grupo clonidina e 38 no grupo placebo, com predomínio do sexo masculino em ambos os grupos $(61 \%$ no grupo clonidina e $76 \%$ no grupo placebo; $p=0,17$ ).

A média de peso foi de $7,2 \pm 3 \mathrm{~kg}$ no grupo clonidina e de $7 \pm 3 \mathrm{~kg}$ no grupo placebo. A mediana da idade foi 21,5 meses (IQ 7,6 a 36 meses) no grupo clonidina e de 13,5 meses (IQ 7,3 a 26,2 meses) no grupo placebo. Nos demais parâmetros avaliados, também não foram encontrados diferenças entre os grupos (Tabela 2). Em relação à indicação de ventilação mecânica, houve um predomínio de doenças respiratórias em ambos os grupos (clonidina $68 \%$ versus $71 \%$ no grupo placebo; $p=0,3$ ).

A mortalidade observada foi de $16 \%$ (5 casos) no grupo clonidina e 7,9\% (3 casos) no grupo placebo ( $p=$ $0,4)$. Além dos oito pacientes que evoluíram ao óbito, houve exclusão de um paciente em cada grupo (por solicitação do médico assistente e outra por hipotensão refratária). Portanto completaram o estudo 59pacientes, 25 no grupo clonidina e 34 no placebo.

A ocorrência de abstinência em ambos os grupos foi alta (72\% no clonidina e $75 \%$ no placebo) não se observando diferença significativa $(p=0,8)$ nessa prevalência com a adição de clonidina ao esquema de sedação nas crianças submetidas à ventilação mecânica. A mediana do tempo de VM no grupo clonidina foi de 7 dias (IQ25\%-75\% - 7 a 10 dias), enquanto que no grupo placebo foi de 6 dias (IQ25\%-75\% - 5 a 10 dias) sem diferença entre eles (Tabela 2 e Figura 1).

Tabela 2 - Características Gerais dos Grupos Clonidina e Placebo

\begin{tabular}{|c|c|c|c|}
\hline Variáveis & Clonidina $(n=31)$ & Placebo $(n=38)$ & $\mathrm{p}$ \\
\hline Sexo & & & 0,2 \\
\hline Feminino & 12 & 9 & \\
\hline Idade (meses) ** & $21,5(7,6-36)$ & $13,5(7,3-26,2)$ & 0,3 \\
\hline Indicação de ventilação mecânica (n e \%) & & & 0,3 \\
\hline Doença respiratória & $21(68)$ & $27(71)$ & \\
\hline Causas de exclusão (n e \%) ${ }^{\star \star *}$ & $1(3,2 \%)$ & $1(2,6 \%)$ & \\
\hline Abstinência (57) £ & $18(72 \%)$ & $24(75 \%)$ & 0,8 \\
\hline Início da abstinência ** (dias) & $0(0-1)$ & $0(0-1)$ & 0,6 \\
\hline Duração da abstinência ** (dias) & $3(0-10)$ & $4(0-10)$ & 0,6 \\
\hline Tempo de VM ${ }^{* \star} £$ (dias) & $7(7-10)$ & $6(5-10)$ & 0,4 \\
\hline
\end{tabular}

* Valores expressos em média \pm DP

** Mediana e intervalo interquartis (P25\%-75\%)

*** hipotensão refratária e solicitação do médico assistente.

$£$ Refere-se aos 59 pacientes vivos que completaram todo o estudo (clonidina $=25$ e placebo $=34$ ) 


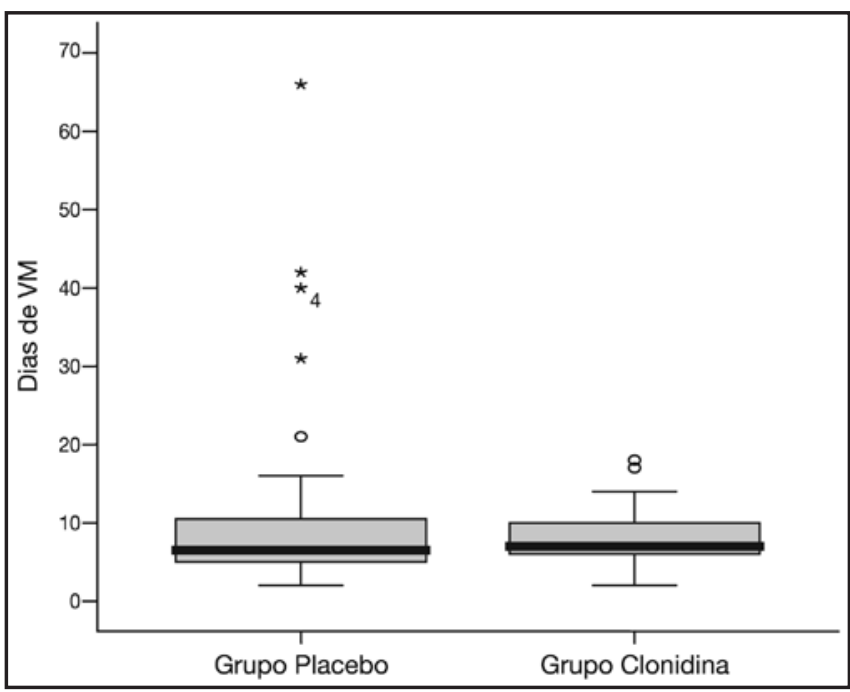

Figura 1 - Tempo de Ventilação Mecânica nos Grupos Estudados
A adição de clonidina ou placebo por via enteral,no início da utilização de morfina e midazolam em infusão contínua para crianças submetidas à VM, não influiu significativamente nas doses diárias ou nas doses cumulativas das medicações sedativas e analgésicas prescritas pelos médicos ao longo do estudo. Também não se observou diferença na utilização de outras medicações para sedação, como diazepam, hidrato de cloral, cetamina e pancurônio, como se observa na figura 2.

As infusões contínuas de midazolam e morfina, assim como as curvas de acompanhamento diário não diferiram entre os grupos. Observou-se que as curvas foram paralelas e superponíveis, tanto na administração de midazolam quanto na de morfina em ambos os grupos (Figura 3).
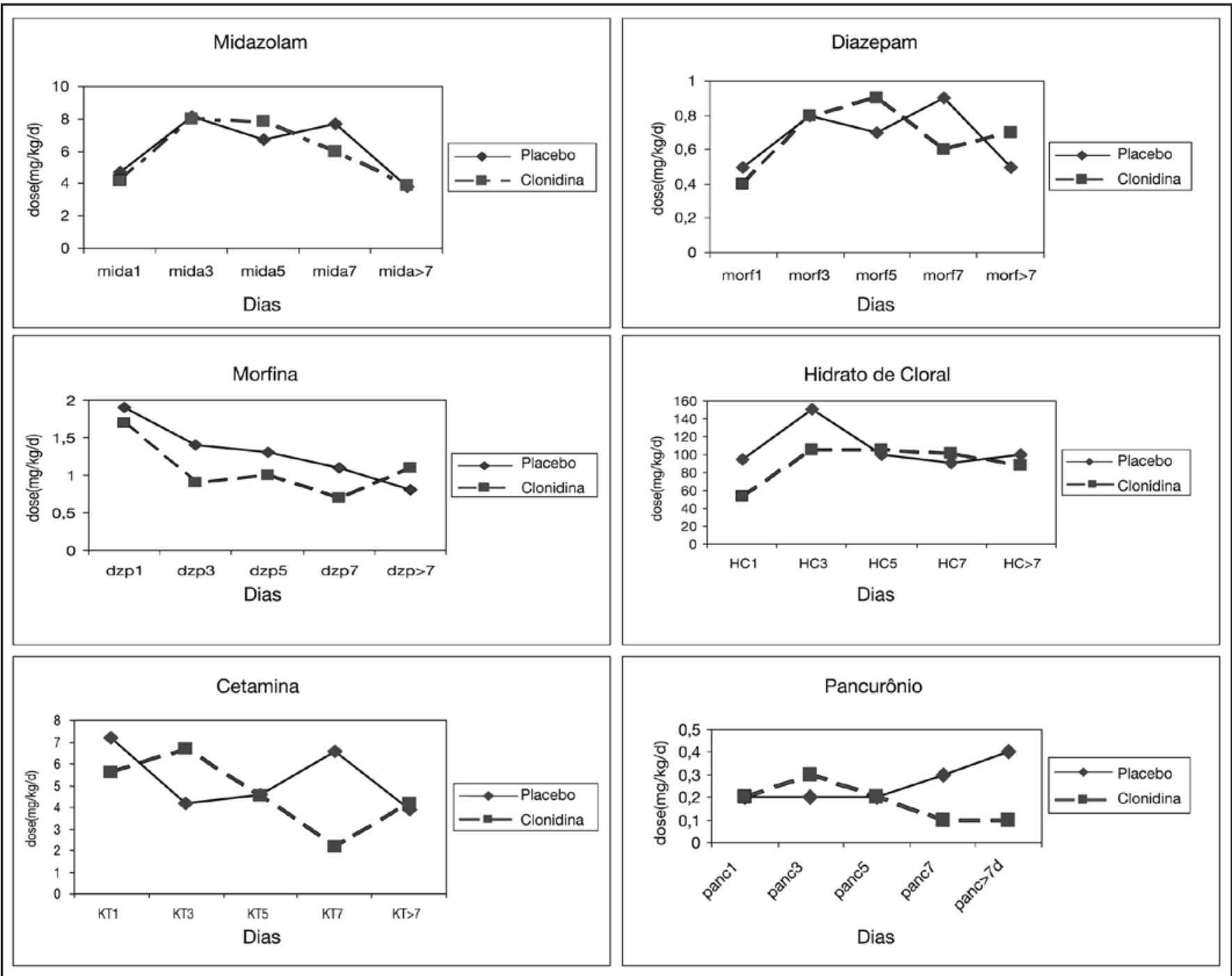

Figura 2 - Comparações nas Doses de Midazolam, Morfina, Diazepam, Hidrato de Cloral, Cetamina e Pancurônio nos dias 1, 3, 5,7 e acima de 7 Dias de Infusão Contínua entre os Grupos Estudados. 


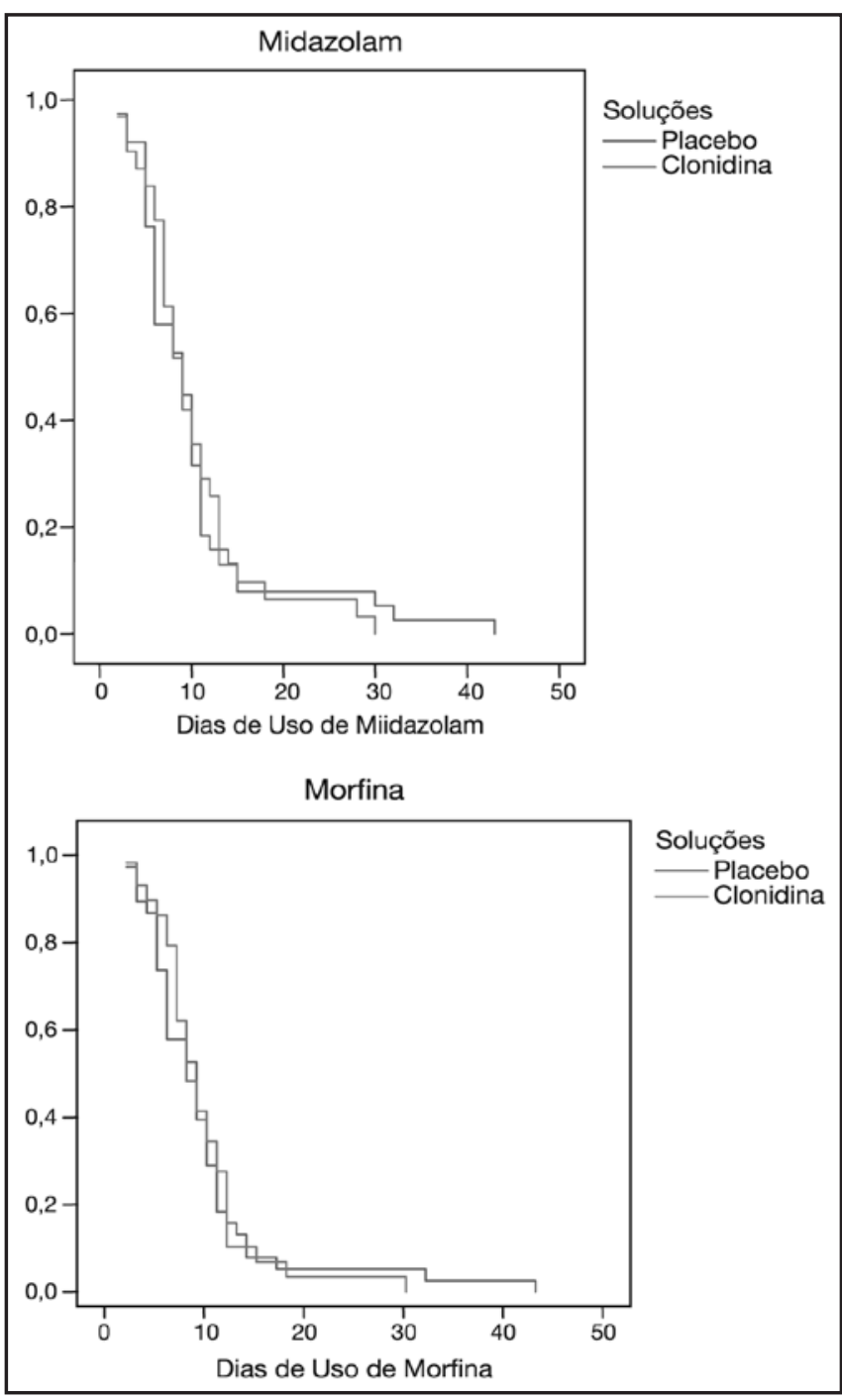

Figura 3 - Comparações nas Infusões Diárias de Midazolam e Morfina entre os Grupos Estudados

A adição de clonidina ao protocolo de sedação de crianças em ventilação mecânica não influiu nas doses cumulativas totais de midazolam e morfina, quando comparada com o placebo.

O tempo de início da abstinência em relação à suspensão da infusão contínua, em ambos os grupos, concentrou-se na data da suspensão (IQ - zero a 1 dia) (Tabela 2). O tempo de resolução da abstinência também não diferiu nos 2 grupos: no grupo clonidina a mediana foi 3 dias (IQ - zero a 10 dias) e, no grupo placebo, de 4 dias (IQ - zero a 10 dias) conforme mostra a tabela 2.

\section{DISCUSSÃO}

Este estudo comparou a administração, por via ente- ral de clonidina ou placebo em crianças submetidas à VM que utilizavam infusão contínua de sedativos e analgésicos em altas doses, constatando que a adição de clonidina não repercutiu nas doses diárias e nas doses cumulativas em infusão contínua de midazolam e morfina; no tempo de uso de midazolam e morfina; na necessidade de outros sedativos como diazepam, hidrato de cloral, cetamina e o uso de bloqueadores neuromusculares; na prevalência de abstinência; no tempo entre a suspensão da infusão contínua e o início dos sintomas de abstinência e na duração da síndrome de abstinência.

A ineficácia apresentada pela clonidina, por via enteral, neste estudo, em potencializar os efeitos sedativos em crianças submetidas à VM, poderia ser atribuída a:

a) Não efetividade do fármaco: vários estudos já demonstraram a eficácia da clonidina, tanto como sedativos $^{2,4,7,18,20,21-28}$, como no tratamento da abstinência ${ }^{2-}$ 4,5,9,12,16,17,19,29. Em alguns estudos, quando utilizada para sedação leve, a clonidina teve resultados comparáveis ao fentanil, evitando doses extras de sedativos ${ }^{21}$. Em apenas um estudo a clonidina e o placebo não apresentaram diferenças ${ }^{6}$. Porém deve-se ressaltar que neste estudo, as doses de clonidina foram baixas $(0,625$ e $1,25 \mu \mathrm{g} / \mathrm{kg})$, quando comparadas às doses preconizadas na literatura. Portanto, quando o objetivo é sedação leve a moderada, a quase totalidade de estudos prévios tem demonstrado que a clonidina tem ação eficaz;

b) Doses inadequadas: na maioria dos estudos as doses de clonidina utilizadas variaram de 2 a $6 \mu \mathrm{g} / \mathrm{kg} /$ dose $e^{5,7,28,30}$, sendo que administração de 2 a $3 \mu \mathrm{g} / \mathrm{kg} /$ dose tem menor efetividade ${ }^{28}$ quando comparada a doses maiores de 4 a $6 \mu \mathrm{g} / \mathrm{kg} / \mathrm{dose}^{5,7,30}$. Portanto, a dose utilizada neste estudo ( $5 \mu \mathrm{g} / \mathrm{kg}$ a cada $8 \mathrm{~h})$ segue as recomendações, devendo ser consideradas como adequada e efetiva;

c) Via de administração inadequada: a maior parte dos estudos emprega a clonidina por via oral, havendo também relatos da utilização por via venosa, seja em doses intermitentes ou em infusão contínua ${ }^{18,19}$. Quando da utilização em infusão contínua, as doses têm oscilado entre 0,3 e 0,8 $\mu \mathrm{g} / \mathrm{kg} / \mathrm{h}^{18,19}$. A clonidina é bem absorvida por via oral, atingindo uma biodisponibilidade de quase $100 \%$. Observa-se o efeito máximo em 1 a 3 horas, com um pico de efeito sedativo em 48 a 72 horas $^{4,5,10,11,23}$. Dessa forma, não se poderia creditar a utilização da via oral como o fator determinante para o insucesso da clonidina neste estudo;

d) Interação com outras medicações: na terapia inten- 
siva pediátrica, com freqüência, são utilizadas várias medicações, tais como sedativos bloqueadores dos receptores $\mathrm{H}_{2}$, anticonvulsivantes e antibióticos, que podem interagir com a clonidina. Sabe-se que podem ocorrer interações medicamentosas que comprometam a absorção, o metabolismo, a distribuição e o bloqueio no local de ação ${ }^{10,11}$. Por outro lado, a clonidina também atua nos receptores imidazólicos potencializando o efeito dos inibidores do ácido gamabutírico, aumentando o efeito sedativo e hipotensor ${ }^{11}$. Fato este que favoreceria a maior ação sedativa no grupo que recebeu clonidina por via oral. Entretanto, a interação com outros medicamentos, fator que poderia influir na ação da clonidina, não foi avaliada neste estudo.

e) Desfecho - sedação excessiva: é prática comum em UTIP a manutenção de crianças em VM com sedação mais profunda, inibindo, inclusive qualquer movimentação voluntária ${ }^{1}$. Essa estratégia que, em um primeiro momento parece facilitar a ventilação mecânica, tem como desvantagem aumentar o tempo de ventilação, o tempo de internação na UTI e conseqüentemente a morbidade associada ${ }^{1,2,29}$. Não existem dúvidas que, para manter o paciente absolutamente sedado, faz-se necessário o emprego de maiores doses intermitentes de sedativos, assim como o aumento nas infusões de morfina e midazolam ${ }^{1,2,29}$. Alternativamente, tem sido proposto que os pacientes em VM sejam mantidos mais alertas, interagindo com o meio e preservando a ventilação espontânea ${ }^{1,31}$. Na escala de sedação de Ramsay, esse estágio é representado por escore entre 2 e $3^{20}$.

Existem evidências demonstrando que neste estudo as crianças submetidas à VM tenham sido excessivamente sedadas, tais como: as altas doses de morfina (doses diárias de aproximadamente $0,85 \mathrm{mg} / \mathrm{kg} / \mathrm{dia}$ ), de midazolam (doses diárias de $8 \mathrm{mg} / \mathrm{kg} / \mathrm{dia}$ ), e de sedativos intermitentes, assim como o seu prolongado tempo de uso (mediana 9 dias, tanto para morfina como midazolam), que é muito acima do recomendado na literatura ${ }^{1,2,5,33}$.

Sabe-se que a utilização de fentanil em doses cumulativas de 1,6 a $2,5 \mathrm{mg} / \mathrm{kg}$ e por tempo acima de 5 a 9 dias, assim como o midazolam em doses superiores a $60 \mathrm{mg} / \mathrm{kg}$, pelo mesmo período, estão relacionadas ao aparecimento de abstinência ${ }^{3,9}$. As doses de morfina relacionadas ao desenvolvimento da abstinência não estão bem estabelecidas ${ }^{3}$, já que há maior afinidade dos opióides sintéticos com os receptores do que com os não sintéticos. Também a ocorrência de tolerância e abstinência está relacionada com o tempo de ligação dos fármacos aos receptores e as doses utilizadas. O uso de altas doses de sedativos em nosso meio já foi descrito por Sfoggia e col. ${ }^{1}$, que verificou a utilização de doses médias de midazolam $(0,54 \pm 0,3 \mu \mathrm{g} / \mathrm{kg} / \mathrm{h})$, de morfina $(22,2 \pm 17 \mu \mathrm{g} / \mathrm{kg} / \mathrm{h})$ e observaram prevalência de abstinência de $34,1 \%$. Outros estudos também demonstraram a utilização de altas doses de sedativos com o objetivo de facilitar a ventilação ${ }^{24}$.

É interessante ressaltar que a clonidina quando associada a baixas doses de sedativos analgésicos, pode prevenir ou reduzir o aparecimento de abstinência ${ }^{4,9,15,29}$. Entretanto, neste estudo sugere-se que a clonidina perde essa ação preventiva quando utilizada em conjunto com altas doses de sedativos, pois a prevalência em ambos os grupos foi acima de $70 \%$, muito superior ao descrito na literatura, em torno de $35 \%$ e $57 \%^{1,9,15,29,33}$. Portanto, parece que a clonidina para apresentar seus efeitos sedativos e protetores de abstinência, deve ser utilizada em associação com baixas doses de opióides e diazepínicos.

Com o intuito de promover boa sedação e mínima abstinência, novos sedativos têm sido preconizados. Dentre as medicações utilizadas com essa finalidade está a clonidina. A sedação excessiva é um erro freqüente, e com o intuito de reduzir a tolerância e a abstinência, alguns estudos têm proposto a interrupção diária da infusão contínua, com reinício subseqüente, em doses menores ${ }^{15,30}$. Outra estratégia é a utilização de menores doses de sedativos e analgésicos, objetivando sedação menos profunda, mantendo um estado de sonolência e responsivo, com escala de Ramsay entre 2 e $3^{20}$. É dentro desse cenário que a clonidina pode apresentar sua máxima eficácia de sedação e prevenção de abstinência.

\section{REFERÊNCIAS}

1. Sfoggia A, Fontela PS, Moraes A et al - Sedation and analgesia in children submitted to mechanical ventilation could be overestimated? $\mathrm{J} \mathrm{Pe}$ diatr, 2003;79:343-348.

2. 02. Jacobi J, Fraser GL, Coursin DB et al - Clinical practice guidelines for sustained use of sedatives and analgesics in the critically ill adult. Crit Care Med, 2002;30:119-141.

3. 03 . Kesavulu V, Costarino AT - Pediatric sedation withdrawal: Recognizing the need for refinement in a necessary therapy. Crit Care Med, 1999;27:32-33.

4. $\quad$ 4. Knight G, Ramelet AS, Duncan A et al - Analgesia e Sedação em UTIP, em: Piva JP, Garcia PCR - Medicina Intensiva em Pediatria. Rio de Janeiro: Revinter, 2005;733-752.

5. 05. Tobias JD - Tolerance, withdrawal, and physical dependency after long-term sedation and analgesia of children in the pediatric intensive care unit. Crit Care Med, 2000;28:2122-2132

6. 06. Freeman KO, Connelly NR, Schwartz D et al - Analgesia for paediatric tonsillectomy and adenoidectomy with intramuscular clonidine. Paediatr Anaesth, 2002;12:617-620.

7. 07 . Fujii $\mathrm{Y}$, Saitoh $\mathrm{Y}$, Tanaka $\mathrm{H}$ et al - Pretreatment with oral clonidine 


\section{CLONIDINA ASSOCIADA À MORFINA E MIDAZOLAM EM CRIANÇAS SUBMETIDAS À VENTILAÇÃO MECÂNICA: ESTUDO ALEATÓRIO, DUPLAMENTE ENCOBERTO E PLACEBO CONTROLADO}

attenuates cardiovascular responses to tracheal extubation in children. Paediatr Anaesth, 2000;10:65-67.

8. 08. Gowing L, Farrell M, Ali R et al - Alpha2 adrenergic agonists for the management of opioid withdrawal. Cochrane Database Syst Rev, 2004;18 (4):CD002024, DOI: 10.102/14651858.

9. 09. Suresh S, Anand KJ - Opioid tolerance in neonates: a state-of-the-art review. Paediatr Anaesth, 2001;11:511-521.

10. 10 Hoffman BB, Lefkowitz RB - Catecolaminas, Drogas Simpaticomiméticas e Antagonistas dos Receptores Adrenérgicos, em: Goodman Gilman A, Hardman JG, Limbird LE et al - As bases Farmacológicas da Terapêutica. Rio de Janeiro: McGraw-Hill, 1996;146-182.

11. Seger DL - Clonidine toxicology revisited. J Toxicol Clin Toxicol, 2002;40:145-155

12. Miser AW, Chayt KJ, Sandlund JT et al - Narcotic withdrawal syndrome in young adults after the therapeutic use of opiates, Am J Dis Child, 1986;140:603-604.

13. Zarrindast MR, Homayoun $\mathrm{H}$, Khavandgar $\mathrm{S}$ et al - The effects of simultaneous administration of alpha2-adrenergic agents with L-NAME or $\mathrm{L}$-arginine on the development and expression of morphine dependence in mice. Behav Pharmacol, 2002;13:117-125.

14. Finkel JC - Opioid tolerance and dependence in infants and children http://www.pedsanesthesia.org/meetings/2004winter/pdfs/finkel Refreshercourse.pdf acessado em 22/12/2006

15. Cunliffe M, McArthur L, Dooley F - Managing sedation withdrawal in children who undergo prolonged PICU admission after discharge to the ward. Paediatr Anaesth, 2004;14:293-298.

16. Tobias JD, Deshpande JK, Gregory DF - Outpatient therapy of iatrogenic drug dependency following prolonged sedation in the pediatric intensive care unit. Intensive Care Med, 1994;20:504-507.

17. Osborn DA, Jeffery HE, Cole MJ - Sedatives for opiate withdrawal in newborn infants. The Cochrane Database Syst Rev, 2005;20.CD 002053 , DOI 10.1002/14651858.

18. Ambrose C, Sale S, Howells R et al - Intravenous clonidine infusion in critically ill children: dose-dependent sedative effects and cardiovascular stability. Br J Anaesth, 2000;84:794-796.

19. Lowery R, Zuk J, Polaner DM - Long-term use of clonidine in a criticallyill infant. Paediatr Anaesth, 2005;15:694-698.
20. Crean P - Sedation and neuromuscular blockade in paediatric intensive care; practice in the United Kingdom and North America. Paediatr Anaesth, 2004;14:439-442.

21. Reimer EJ, Dunn GS, Montgomery CJ et al - The effectiveness of clonidine as an analgesic in paediatric adenotonsillectomy. Can J Anaesth, 1998;45:1162-1167

22. Nishina K, Mikawa K, Shiga M et al - Clonidine in paediatric anaesthesia. Paediatr Anaesth, 1999;9:187-202.

23. Cigada M, Pezzi A, Di Mauro $P$ et al - Sedation in the critically ill ventilated patient: possible hole of enteral drugs. Intensive Care Med, 2005;31:482-486.

24. Marx CM, Rosenberg DI, Ambuel B et al - Pediatric intensive care sedation: survey of fellowship training programs. Pediatrics, 1993;91:369 378.

25. Lundeberg S, Lonnqvist PA - Update on systemic postoperative analgesia in children. Paediatr Anaesth, 2004;14:394-397.

26. Ambrose C, Sale S, Howells R et al - Intravenous clonidine infusion in critically ill children: dose-dependent sedative effects and cardiovascular stability. Br J Anaesth, 2000;84:794-796.

27. Wiley JF $2^{\text {nd }}$, Wiley CC, Torrey SB et al - Clonidine poisoning in young children. J Pediatr, 1990;116:654-658.

28. Ozdogan UK, Lahdesmaki J, Hakala K et al - The involvement of alpha 2A-adrenoceptors in morphine analgesia, tolerance and withdrawal in mice. Eur J Pharmacol, 2004;497:161-171.

29. Bicudo JN, de Souza N, Mangia CM et al - Withdrawal syndrome associated with cessation of fentanyl and midazolam in pediatrics. Rev Assoc Med Bras, 1999;45:15-18.

30. Kress JP, Pohlman AS, O'Connor MF et al - Daily Interruption of sedative infusions in critically ill patients undergoing mechanical ventilation. N Engl J Med, 2000;342:1471-1477.

31. Bohrer $\mathrm{H}$, Schmidt $\mathrm{H}$, Bach $\mathrm{A}$ et al - Methadone treatment of opioid withdrawal in intensive care patients. Lancet, 1993;341:636-637.

32. Polaner DM - Sedation-analgesia in the pediatric intensive care unit. $\mathrm{Pe}-$ diatr Clin North Am, 2001;48:695-711.

33. Bartolome SM, Cid JLH, Freddi N - Sedação e analgesia em crianças: uma abordagem prática para situações freqüentes. J Pediatr, 2007;83: S71-S82. 